\title{
Qualité microbiologique et physico-chimique des laits fermentés consommés à Maroua (Cameroun)
}

\author{
J. MAÏWORE ${ }^{1 *}$, M. P. BAANE ${ }^{2}$, L. Tatsadjieu NGOUNE ${ }^{3}$, J. A. FADILA ${ }^{1}$, \\ M. Yaouba YERO ${ }^{1}$ et D. MONTET ${ }^{4}$ \\ ${ }^{l}$ Département des Sciences de la Vie et de la Terre, Université de Maroua, Ecole Normale Supérieure de \\ Maroua. BP 55, Maroua, Cameroun. \\ ${ }^{2}$ Laboratoire de l'Hôpital de la Caisse Nationale de Prévoyance Sociale (CNPS), BP 120 Maroua, Cameroun. \\ ${ }^{3}$ Département des Sciences Alimentaires et Nutrition, Ecole Nationale Supérieure des Sciences Agro- \\ Industrielles, Université de Ngaoundéré, BP 454, Ngaoundéré, Adamaoua, Cameroun. \\ ${ }^{4}$ Département des Performances des systèmes de production et de transformation tropicaux. UMR 95 \\ QUALISUD. Maison de la Technologie TA B-95/16, 73, rue JF Breton 34398 Montpellier Cedex 5. \\ *Corresponding author; E-mail: maiworejustine@yahoo.fr
}

\section{RESUME}

Le non-respect des règles d'hygiène au cours de la production des laits fermentés peut quelquefois induire une contamination des produits par des microorganismes potentiellement pathogènes. C'est dans cette optique qu'une étude a été réalisée dans le but d'évaluer la qualité physico-chimique et microbiologique des laits fermentés consommés dans la ville de Maroua (Cameroun). La méthode de titration a été utilisée pour les paramètres physico-chimiques et la technique de culture sur boites de pétri a été utilisée pour les analyses microbiologiques. Les résultats obtenus au cours des analyses physico-chimiques ont révélé que les $\mathrm{pH}$ des échantillons sont compris entre 3,4 pour les laits fermentés non conditionnés (LFNC) et 4,16 pour les conditionnés (LFC). L'acidité titrable des différents échantillons oscillait entre 49 et 227,33 ${ }^{\circ} \mathrm{D}$. Les analyses microbiologiques ont révélé que la flore totale est comprise entre $0,57 \times 10^{4}$ et $1,72 \times 10^{6} \mathrm{UFC} / \mathrm{ml}$; la flore lactique est comprise entre 0 et $1 \times 10^{7} \mathrm{UFC} / \mathrm{ml}$ (LFNC8); la flore fongique est comprise entre 0 et $1 \times 10^{7}$ $\mathrm{UFC} / \mathrm{ml}$; les coliformes totaux ont une concentration comprise entre 0 et $5,76 \times 10^{4} \mathrm{UFC} / \mathrm{ml}$; les coliformes fécaux sont compris entre 0 et $2,70 \times 10^{3}$ avec $44 \%$ d'échantillons de laits fermentés contaminés par ces bactéries. Les salmonelles n'ont pas été détectées dans les échantillons analysés. Dans l'ensemble (100\%), la qualité des différents laits fermentés analysés n'est pas satisfaisante par rapport aux coliformes fécaux et totaux ; la flore fongique, la flore lactique et la flore totale.

(C) 2018 International Formulae Group. All rights reserved.

Mots clés: Laits fermentés, qualité microbiologique, $\mathrm{pH}$, acidité, contamination.

\section{Microbiological and physico-chemical quality of fermented milks consumed in Maroua (Cameroun)}

\begin{abstract}
The non respect of hygiene regulation during the production of fermented milks could sometimes lead to a contamination of products by potentially pathogenic microorganisms. It is from this perspective that a study was realized with the aim of assessing the physico-chemical and microbial quality of fermented milk consumed
\end{abstract}


in the city of Maroua (Cammeroon). The method of titration was used for the physico-chemical parameters and the technique of culture on petri dishes was used for the microbiological analyses. The results obtained during the physico-chemical analyses revealed that the $\mathrm{pH}$ of the samples were between 3,4 for fermented not packed milk (LFNC) and 4,16 for the packed one (LFC). The titrable acidity of the various samples was between 49 and $227.33^{\circ} \mathrm{D}$. The microbiological analyses revealed that the total flora was between $0.57 \times 10^{4}$ and $1.72 \times 10^{6}$ $\mathrm{UFC} / \mathrm{ml}$; the lactic acid flora was between 0 and $1 \times 10^{7} \mathrm{UFC} / \mathrm{ml}$ (LFNC8); the fungal flora was between 0 and $1 \times 10^{7} \mathrm{UFC} / \mathrm{ml}$; the concentration of total coliformes was between 0 and $5.76 \times 10^{4} \mathrm{UFC} / \mathrm{ml}$; the faecal coliformes was between 0 and $2.70 \times 10^{3}$ with $44 \%$ of the samples of fermented milk contaminated by these bacteria. Salmonellas were not detected in the analysed samples. In the majority of the cases, the quality of various analyzed fermented milks was not satisfactory.

(c) 2018 International Formulae Group. All rights reserved.

Keywords: Fermented milks, microbial quality, pH, titrable acidity, contamination.

\section{INTRODUCTION}

La région du Nord du Cameroun a une culture séculaire basée sur la consommation du lait (Essomba et Dury, 2000 ; Monkam et al., 2002). En général, après la traite, la production locale du lait frais de vache peut être utilisée de diverses manières. Le lait ainsi obtenu après la traite peut être vendu tel quel, soit à des personnes qui le font juste bouillir pour une consommation domestique, soit à des producteurs locaux qui vont le transformer.

Dans cette partie du pays appartenant à la zone septentrionale du Cameroun, la température ambiante élevée peut atteindre $42^{\circ} \mathrm{C}$. L'insuffisance des systèmes réfrigérés a contraint les populations à transformer le lait pour éviter sa dégradation. De plus, la teneur en eau et en nutriments du lait font de lui une denrée alimentaire hautement périssable qui favorise la croissance de microorganismes tels que les levures, les moisissures et les bactéries (Hamad, 2009; Dadie, 2010 ; Anses, 2015).

Dans l'optique d'augmenter sa durée de conservation, le lait peut subir de nombreuses transformations (Vignola, 2002) et donner ainsi lieu à différents produits tels que : le "Pendidam" (lait fermenté écrémé), le "Kidirmou" (lait fermenté entier), le yaourt, le beurre ou le fromage. Les laits fermentés produits sont conditionnés ou conservés dans des calebasses et déposés soit dans des alimentations ou épiceries pour les premiers, soit dans les marchés environnants, pour les seconds, afin d'être commercialisés. Dans les épiceries, les produits sont conservés dans des réfrigérateurs par contre au marché, le lait caillé ou fermenté est maintenu à température ambiante dans des calebasses. Cependant, les produits laitiers en général et les laits fermentés en particulier doivent être bien conservés tout au long de la chaîne, que ce soit chez le producteur, le distributeur ou le consommateur pour éviter d'éventuelles contaminations (Broutin et al., 2005). Les producteurs des laits fermentés de la ville de Maroua conservent leurs produits dans des réfrigérateurs ou des congélateurs. Quant-aux consommateurs, la conservation n'est pas toujours bien réalisée, certains utilisent des réfrigérateurs, d'autres par contre conservent leurs produits laitiers à température ambiante pendant plusieurs heures ou jours, ce qui favorise une contamination par des microorganismes (Essomba, 2000).

$\mathrm{La}$ seule structure industrielle qui produisait hygiéniquement le yaourt dans le Nord était le projet laitier pilote de Ngaoundéré. Né d'un financement entre le Canada et la Cameroun en 1994, ce projet avait pour mission de développer la production de lait et d'encourager la consommation de produits laitiers dans l'Adamaoua et toute la zone nord du pays. Il a, malheureusement, fermé ses portes quelques années plus tard en 2002. Cette zone du pays a ainsi connu une prolifération de producteurs artisanaux de yaourts. On a dès lors, assisté à une production de yaourts dont la qualité n'est pas contrôlée. Des enquêtes menées auprès des consommateurs ont révélé que de nombreux cas de diarrhée ont été 
décelés. Il n'est pas rare de constater que certains pots explosent littéralement au visage des consommateurs lors de l'ouverture du bouchon. Tout laisse à croire que la flore présente dans les yaourts incriminés n'est pas constituée uniquement de bactéries lactiques.

De nombreux travaux tels que ceux de Coudeyras et Forestier (2010), Heyman et Heuvelin (2006), Joly et al. (2007), Drouault (2001), Savadogo et Traore (2011) ont montré que l'administration de laits fermentés, dont le yaourt, tend à rétablir un équilibre bactérien favorable à la normalisation du transit digestif. Puisque le lait fermenté et le yaourt peuvent permettre de rétablir le transit digestif et, quand on sait que ces produits sont fabriqués en grande quantité, de façon artisanale et non contrôlée, il devient donc impératif d'évaluer la qualité microbiologique des laits fermentés et des yaourts produits dans cette ville. Le but de cette étude a donc été de déterminer quelques paramètres physico-chimiques et d'évaluer les qualités microbiologiques des laits fermentés commercialisés dans la ville de Maroua au Cameroun.

\section{MATERIEL ET METHODES}

\section{Zone d'étude et échantillonnage}

Les échantillons ont été prélevés dans la ville de Maroua, Région de l'Extrême-Nord Cameroun plus précisément dans le Département du Diamaré de janvier à avril 2015. Cette Région jouit d'un environnement favorable au développement de l'élevage, le lait et ses dérivés font ainsi partie intégrante de l'alimentation. Le lait produit par les vaches sert à la consommation domestique et le surplus est quelquefois vendu pour subvenir aux besoins des familles d'éleveurs. Parmi les produits laitiers les plus consommés figurent : le yaourt (c), le "kindirmou" (a) et le "pendidam" (b) (Figure 1). En général, la technique de fabrication utilisée par les producteurs de laits fermentés se fait en plusieurs étapes (Figure 2). Le lait produit peut être transformé par les épouses d'éleveurs et les femmes Peuhl en "kindirmou" ou en "pendidam". Le "kindirmou" est un type de lait fermenté produit à partir du lait de vache, chauffé puis caillé et conservé dans des calebasses appropriées (Essomba et Dury, 2002). Le kindirmou a un goût acide, a l'aspect d'un yaourt étuvé et est consommé simplement après ajout de sucre. Le "pendidam", quant à lui, désigne le lait fermenté acide, obtenu à partir d'un lait frais bouilli puis fermenté grâce à un lait fermenté issu d'une précédente production (Figure 2). A la différence du "kidirmou", le "pendidam" est moins riche en matière grasse mais les deux sont très proches sur le plan technologique et microbiologique. Les deux laits produits ne sont point réfrigérés mais conservés à température ambiante pendant plusieurs jours. Le pendidam peut aussi être consommé tel qu'il est, mais dans la plupart des cas, il est ajouté à des bouillies en fin de cuisson pour l'acidifier. Le lait fermenté étiqueté (yaourt sur la Figure 2) est produit par des producteurs artisanaux qui utilisent soit le lait en poudre (dans la plupart des cas), soit le lait frais. A la fin, les produits obtenus sont conservés dans des réfrigérateurs et quelquefois dans des congélateurs.

$\mathrm{Au}$ total, 54 échantillons de laits fermentés ont été prélevés chez 18 producteurs des quartiers ci-après de la ville de Maroua: Pitoaré, Dougoy, Diguirwo, Domayo, Baoliwol, Marché Abattoir, Hardé, Doualaré, Palar et Congoré. Ces derniers ont été répartis en 2 lots : le premier lot était constitué des laits fermentés ou yaourts conditionnés et vendus dans les alimentations. Le second lot, quant à lui, était constitué d'échantillons des laits fermentés ("Kidirmou" et "pendidam") produits par les femmes Peuhl dans les calebasses et vendus au marché. Vingt-quatre échantillons fermentés dans les calebasses ont été séparément prélevés dans des flacons en verre stériles de $250 \mathrm{~mL}$ chez les vendeuses. Trente autres échantillons conditionnés dans les flacons en polyéthylène $(250 \mathrm{~mL})$ ont été collectés tels qu'ils se présentaient directement dans les alimentations au moment de la livraison. Les échantillons collectés ont ensuite été placés dans une glacière contenant des carboglaces congelés pour être acheminés au laboratoire. 
Les échantillons ont été analysés le jour même du prélèvement.

\section{Analyses \\ Analyses physico-chimiques : mesure du pH et de l'acidité}

Le $\mathrm{pH}$ a été mesuré par immersion directe de l'électrode du pH-mètre électronique (HANNA, Japon) dans le lait fermenté contenu dans un bécher contenant 20 $\mathrm{ml}$ d'échantillon à $25^{\circ} \mathrm{C}$. L'opération a été répétée trois fois (AFNOR, 2009).

L'acidité a été déterminée par la technique de titration. Pour cela, $10 \mathrm{~mL}$ de lait fermenté ont été prélevés et versés dans un bécher. Trois à 4 gouttes de phénolphtaléine ont été ajoutées au lait sous une constante agitation. La titration a été faite à température ambiante par ajout gouttes à gouttes de la solution de $\mathrm{NaOH}$ de $0,1 \mathrm{~N}$ jusqu'au virage au rose, le volume final de la soude a ainsi été noté (AOAC, 2005). L'acidité a été exprimée en degré Dornic $\left(1^{\circ}\right.$ Dornic correspond à $0,1 \mathrm{~g}$ d'acide lactique). Chaque échantillon a été titré 3 fois. Les critères de qualité physicochimique adoptés lors de cette étude sont tirés de la norme pour les laits caillés citée par Alais (1985).

\section{Analyses microbiologiques}

Les échantillons de lait fermenté ont été homogénéisés puis, $25 \mathrm{~g}$ de chaque échantillon ont été aseptiquement pesés et ajoutés à $225 \mathrm{~mL}$ d'eau peptonnée (Oxoid Cambridge, UK). Après mélange, les dilutions décimales ont été effectuées. Les différentes dilutions $\left(10^{-1}\right.$ à $\left.10^{-6}\right)$ ont été ensemencées en double sur des milieux de culture gélosés et incubés à des températures précises en fonction du microorganisme à dénombrer.

Pour isoler la flore totale, $100 \mu \mathrm{L}$ d'échantillon ont été étalés sur la gélose PCA (Plate Count Agar (Oxoid, Basingstoke, UK). Les boîtes ensemencées ont été incubées dans une étuve à $30{ }^{\circ} \mathrm{C}$ pendant 72 heures et les colonies obtenues ont été dénombrées (AOAC, 1990). Les bactéries lactiques ont été dénombrées après ensemencement de 100 $\mu \mathrm{L}$ de chaque échantillon dilué sur une gélose MRS (Man, Rogosa et Sharpe) additionné de cycloheximide $(0,1 \mathrm{mg} / \mathrm{L})$. Après 48 heures d'incubation à $37^{\circ} \mathrm{C}$ les colonies obtenues ont été dénombrées. Pour le test présomptif des coliformes, $100 \mu \mathrm{L}$ de chaque dilution ont été ensemencés sur la gélose Violet Red Bile Agar (VRBA; Oxoid, Scharleau, Espagne) et les boites ont été incubées à $37{ }^{\circ} \mathrm{C}$ pour les coliformes totaux et $44{ }^{\circ} \mathrm{C}$ pour les coliformes fécaux. Après 24 heures d'incubation, les colonies rouge-pourpres de $0,5 \mathrm{~mm}$ de diamètre et ayant une zone de précipitation ont été dénombrées. Pour confirmation, les colonies sélectionnées ont été transférées dans le bouillon Vert brillant lactose bile à $2 \%$ pour les coliformes totaux ou le bouillon tryptose de Lauryl pour les coliformes fécaux. Ces cultures ont été incubées à $35^{\circ} \mathrm{C}$ pendant 24 à $48 \mathrm{~h}$ pour une éventuelle production de gaz (FDA, 2001). La flore fongique a été dénombrée après ensemencement de $100 \mu \mathrm{L}$ d'échantillon sur la gélose sabouraud (Fluka, Sigma-Aldrich, India) additionée de Chloramphenicol et incubation à $25{ }^{\circ} \mathrm{C}$ pendant 3 à 5 jours (AFNOR, 2002). Pour le dénombrement présomptif de Salmonella spp., $25 \mathrm{~g}$ de lait fermenté ont préalablement été additionnés à $225 \mathrm{~mL}$ d'eau peptonnée tamponnée et le mélange a été incubé à $37{ }^{\circ} \mathrm{C}$ pendant 18 à 24 heures. Un millilitre de cette préculture a ensuite été ajoutée à $100 \mathrm{~mL}$ de bouillon Rappaport-Vassiliadis (Merck, Germany) et Muller Kauffmann. Chaque mélange a ensuite été incubé à $37^{\circ} \mathrm{C}$ pendant $24 \mathrm{~h}$ pour un enrichissement sélectif. A l'aide d'une anse de platine, la précédente préculture a été ensemencée sur la gélose XLD (Oxoid, Basingstoke, England) contenue dans des boîtes de pétri et l'incubation a été faite à $37{ }^{\circ} \mathrm{C}$ pendant $24 \mathrm{~h}$.

\section{Analyses statistiques}

Les moyennes et écarts-types des différentes répétitions ont été obtenus par utilisation du logiciel Microsoft Office Excel 2007. Sur le logiciel STATGRAPHICS centurion 17.1.06., l'ANOVA à un facteur suivi du test de Duncan pour comparer les moyennes des différents échantillons en fonction des producteurs. 


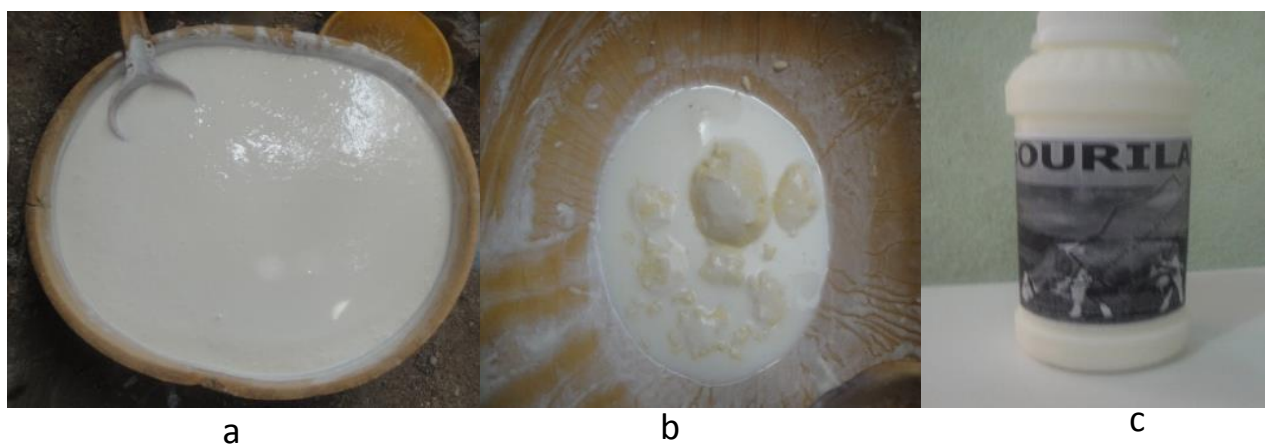

Figure 1: "Kindirmou" (a) "pendidam" (b) dans des calebasses et lait fermenté conditionné appelé yaourt (c).

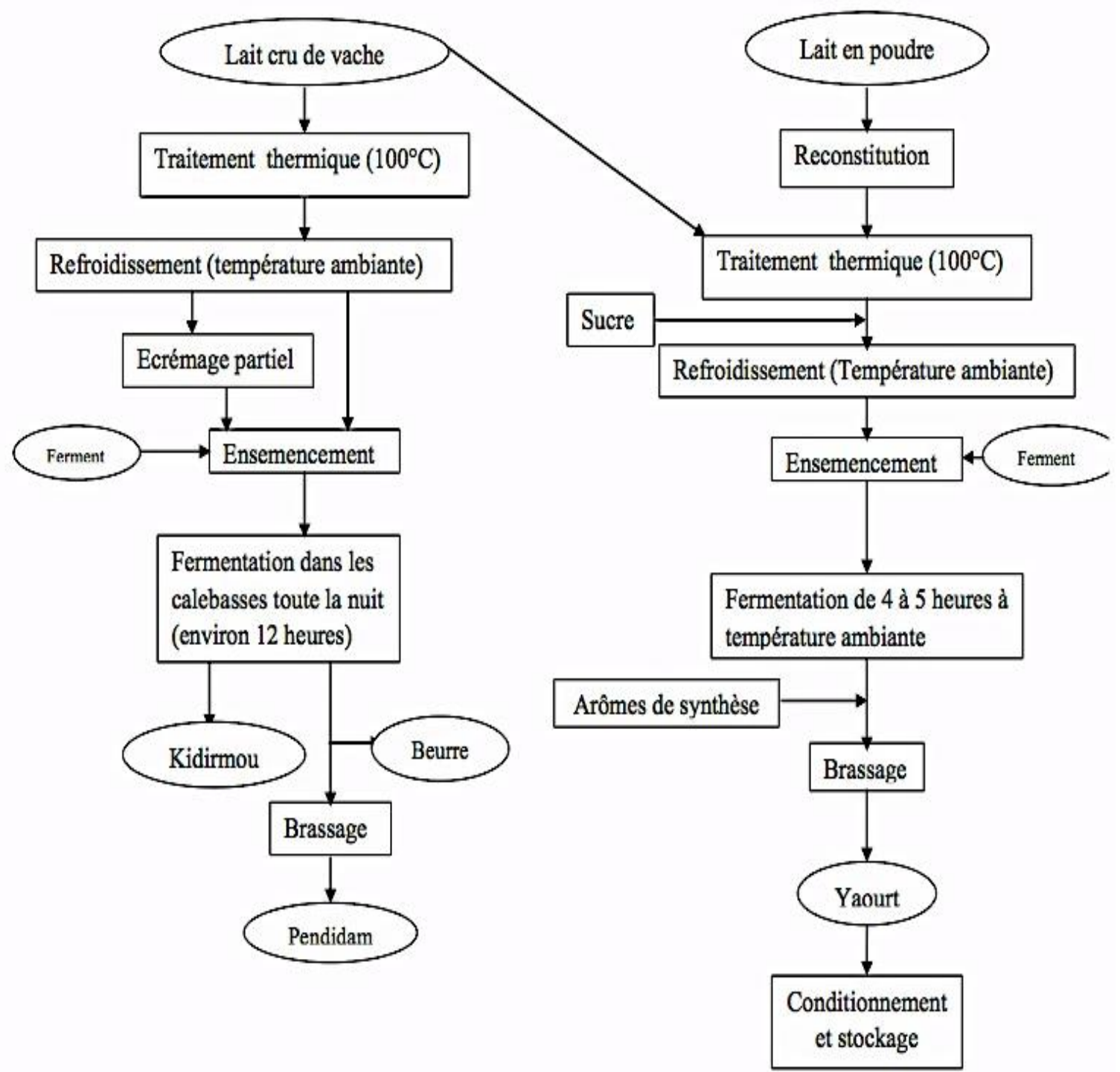

Figure 2 : Diagramme de fabrication des laits fermentés et yaourt dans la ville de Maroua. 


\section{RESULTATS}

\section{PH et acidité titrable des laits fermentés}

Les résultats de $\mathrm{pH}$ des différents échantillons de laits fermentés prélevés sont présentés dans le Tableau 1. L'analyse de ces résultats révèle que le $\mathrm{pH}$ de l'ensemble des échantillons est compris entre 3,4 $\pm 0,1$ (pour LFNC 2) et 4,16 $\pm 0,05$ (LFC1). Les échantillons de laits fermentés non conditionnés (LFNC) possèdent les $\mathrm{pH}$ les plus bas $(3,4)$ tandis que les laits fermentés conditionnés (LFC) possèdent les $\mathrm{pH}$ les plus élevés (4,16). Dans l'ensemble, 100\% d'échantillons ont un pH inférieur à 4,6. L'analyse de variance a permis d'observer une différence statistiquement significative $(\mathrm{p}<0,05)$ entre les moyennes de $\mathrm{pH}$ d'un échantillon à l'autre pour un seuil de $5 \%$.

La mesure de l'acidité titrable des différents échantillons de laits fermentés a permis d'obtenir les résultats consignés dans le Tableau 1. Ceux-ci oscillent entre $49 \pm 1,5$ et $227,33 \pm 2,51^{\circ} \mathrm{D}$. L'analyse de ces résultats révèle que les échantillons de laits fermentés non conditionnés (LFNC) sont les plus acides avec une acidité comprise entre 130,16 $\pm 2,25$ (LFNC5) et 227,33 $\pm 2,51$ (LFNC4) avec $100 \%$ des produits non conditionnés respectant la norme (Acidité titrable $\geq 120^{\circ} \mathrm{D}$ pour les laits caillés). Les laits conditionnés, par contre, ont une acidité plus faible avec des valeurs comprises entre 49 $\pm 1,5$ (LFC9) et $92,83 \pm 2,75$ (LFC5) avec 100\% des produits conditionnés ne respectant pas la norme. L'analyse de variance a révélé une différence statistiquement significative entre les moyennes d'acidités d'un niveau de lait à un autre au seuil de $5 \%$.

\section{Flore microbienne des laits fermentés}

$\mathrm{La}$ flore microbienne des laits fermentés a été dénombrée et les résultats obtenus sont consignés dans le Tableau 2. L'analyse de ces derniers révèle que la concentration microbienne obtenue est fonction $\mathrm{du}$ produit analysé et $\mathrm{du}$ microorganisme recherché. La flore totale est comprise entre $(0,57 \pm 0,41) \times 10^{4}$ et $(1,72 \pm$ $0,18) \times 10^{6} \mathrm{UFC} / \mathrm{ml}$. Les échantillons de laits fermentés non conditionnés présentent de façon significative les taux les plus élevés en flore totale.

Les résultats obtenus révèlent que tous les échantillons de laits fermentés non conditionnés et quelques laits conditionnés (LFC 1, LFC 2, LFC 3, LFC 4 et LFC 6) présentent une flore lactique importante tandis que dans d'autres échantillons, elle n'a pas été détectée. Cette flore est comprise entre 0 et $(1,00 \pm 0,46) 10^{7} \mathrm{UFC} / \mathrm{ml}$ (LFNC8). Exceptés les échantillons LFC5, LFC9, LFC8, LFC7 et LFC10 dans lesquels la flore lactique n'a pas été détectée, tous les autres bien que présentant une charge en bactéries lactiques sont en dessous de la norme prévue par l'AFNOR (nombre de bactéries lactiques supérieur ou égal à $10^{8} \mathrm{UFC} / \mathrm{ml}$ ).

La flore fongique des différents échantillons de lait fermentés analysés est comprise entre 0 et $(1,00 \pm 0,70) \quad x$ $10^{7} \mathrm{UFC} / \mathrm{ml}$. Cette flore est présente sur $90 \%$ des échantillons conditionnés et $50 \%$ d'échantillons non conditionnés. Bien qu'il y'ait moins de laits fermentés non conditionnés contaminés par les levures, il est facile de remarquer que les laits qui en contiennent présentent les concentrations les plus élevées en levures et moisissures. La différence n'est pas statistiquement significative entre tous les autres échantillons exceptés avec les échantillons LFCN2.

Dans quelques échantillons, on note la présence de coliformes totaux et leur concentration est comprise entre 0 et $(5,76 \pm$ $0,76) \times 10^{4} \mathrm{UFC} / \mathrm{ml}$. Tous les échantillons $(100 \%)$ de laits fermentés non conditionnés sont contaminés contre $30 \%$ seulement d'échantillons conditionnés. Dans l'ensemble, $44 \%$ d'échantillons de laits fermentés parmi lesquels et $70 \%$ des laits conditionnés et $0 \%$ des laits non conditionnés ne respectent pas la norme prévue par AFSSA, 2008 sur les yaourts et laits fermentés qui est inférieur à $10 / \mathrm{g}$.

Le Tableau 2 révèle que les coliformes fécaux sont compris entre 0 ou ND et $(2,70 \pm 0,70) \times 10^{3} \mathrm{UFC} / \mathrm{g}$. Ces résultats révèlent également que $44 \%$ d'échantillons de laits fermentés sont contaminés par des 
coliformes fécaux. Parmi ceux-ci, 16,6\% d'échantillons de laits conditionnés (LFC 4, LFC 9 et LFC 10) contre plus de 27,78\% d'échantillons non conditionnés (LFCN 1, LFCN 3, LFCN 6, LFCN 7, LFCN 8) sont contaminés. Ces résultats révèlent également que environ $55 \%$ d'échantillons respectent la norme qui recommande que la concentration en coliformes fécaux soit inférieure à 1 par gramme de produit.

Les salmonelles n'ont pas été détectées dans les échantillons analysés. Ces échantillons sont conformes à la norme prévue par AFNOR en 1999 (absence totale des salmonelles dans les laits fermentés).

Tableau 1: $\mathrm{pH}$ et acidité titrable des laits fermentés conditionnés et non conditionnés.

\begin{tabular}{|c|c|c|c|}
\hline Types de laits fermentés & Echantillons & pH & Acidité $\left({ }^{\circ} \mathbf{D}\right)$ \\
\hline \multirow[t]{10}{*}{ Laits fermentés conditionnés } & LFC1 & $4,16 \pm 0,05^{\mathrm{i}}$ & $78 \pm 2^{\mathrm{de}}$ \\
\hline & LFC 2 & $3,66 \pm 0,05^{\mathrm{de}}$ & $80,83 \pm 2,25^{\mathrm{e}}$ \\
\hline & LFC 3 & $3,56 \pm 0,05^{\mathrm{cd}}$ & $81,66 \pm 1,52^{\mathrm{e}}$ \\
\hline & LFC 4 & $3,66 \pm 0,05^{\mathrm{de}}$ & $60,35 \pm 0,13^{b}$ \\
\hline & LFC 5 & $3,53 \pm 0,05^{\mathrm{bc}}$ & $92,83 \pm 2,75^{\mathrm{g}}$ \\
\hline & LFC 6 & $3,7 \pm 0,1^{\mathrm{ef}}$ & $79,53 \pm 0,05^{\mathrm{de}}$ \\
\hline & LFC 7 & $3,8 \pm 0,1^{\mathrm{fg}}$ & $87,26 \pm 0,25^{\mathrm{f}}$ \\
\hline & LFC 8 & $3,83 \pm 0,05^{\mathrm{gh}}$ & $74,83 \pm 2,25^{\mathrm{d}}$ \\
\hline & LFC 9 & $3,8 \pm 0,1^{\mathrm{fg}}$ & $49 \pm 1,5^{\mathrm{a}}$ \\
\hline & LFC 10 & $3,93 \pm 0,05^{\mathrm{h}}$ & $66 \pm 4,09^{c}$ \\
\hline \multirow[t]{8}{*}{ Laits fermentés non conditionnés } & LFNC 1 & $3,58 \pm 0,01^{\mathrm{cd}}$ & $154,83 \pm 2,75^{\mathrm{j}}$ \\
\hline & LFNC 2 & $3,4 \pm 0,1^{\mathrm{a}}$ & $220,03 \pm 2,55^{\mathrm{m}}$ \\
\hline & LFNC 3 & $3,53 \pm 0,01^{\mathrm{bc}}$ & $195,83 \pm 9,46^{\mathrm{k}}$ \\
\hline & LFNC 4 & $3,46 \pm 0,02^{\mathrm{ab}}$ & $227,33 \pm 2,51^{\mathrm{n}}$ \\
\hline & LFNC 5 & $3,62 \pm 0,02^{\text {cde }}$ & $130,16 \pm 2,25^{\mathrm{h}}$ \\
\hline & LFNC 6 & $3,59 \pm 0,01^{\mathrm{cd}}$ & $142,66 \pm 2,51^{\mathrm{i}}$ \\
\hline & LFNC 7 & $3,62 \pm 0,04^{\mathrm{cde}}$ & $208,33 \pm 2,08^{1}$ \\
\hline & LFNC 8 & $3,57 \pm 0,01^{\mathrm{cd}}$ & $144,16 \pm 3,25^{\mathrm{i}}$ \\
\hline
\end{tabular}

Les valeurs suivies des lettres différentes sont statistiquement différentes $(\mathrm{P}<0,05)$.

LFC : laits fermentés conditionnés

LFNC : laits fermentés non conditionnés 
Tableau 2 : Flore microbienne des laits fermentés conditionnés et non conditionnés.

\begin{tabular}{|c|c|c|c|c|c|c|c|}
\hline $\begin{array}{l}\text { Types de laits } \\
\text { fermentés }\end{array}$ & Echantillons & Flore totale & Bactéries lactiques & Flore fongique & Coliformes totaux & Coliformes fécaux & Salmonella spp. \\
\hline \multirow{9}{*}{ 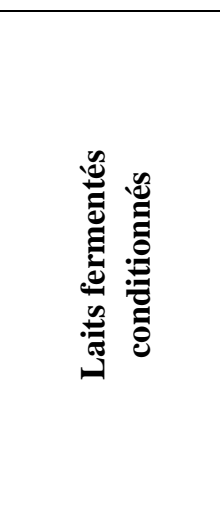 } & LFC1 & $(1,00 \pm 0,05) \times 10^{4 a b}$ & $(5,00 \pm 2,0) \times 10^{4 a}$ & $(0,10 \pm 0,05) \times 10^{4 a}$ & ND & ND & - \\
\hline & LFC 2 & $(0,57 \pm 0,41) \times 10^{4 a}$ & $(1,30 \pm 0,40) \times 10^{5 a}$ & $(8,48 \pm 1,58) \times 10^{4 a}$ & ND & ND & - \\
\hline & LFC 3 & $(2,20 \pm 0,40) \times 10^{5 \mathrm{~d}}$ & $(1,00 \pm 0,25) \times 10^{5 a}$ & $(3,56 \pm 0,38) \times 10^{4 a}$ & $\mathrm{ND}$ & ND & - \\
\hline & LFC 4 & $(1,90 \pm 0,20) \times 10^{5 \mathrm{~cd}}$ & $(8,00 \pm 1,00) \times 10^{4 a}$ & $(0,61 \pm 0,11) \times 10^{4 a}$ & $(1,10 \pm 0,10) \times 10^{3 a}$ & $(1,30 \pm 0,10) \times 10^{4 c}$ & - \\
\hline & LFC 5 & $(5,50 \pm 0,50) \times 10^{5 f}$ & ND & $(0,25 \pm 0,05) \times 10^{4 a}$ & ND & $\mathrm{ND}$ & - \\
\hline & LFC 7 & $(1,10 \pm 0,20) \times 10^{5 b c}$ & ND & $(0,20 \pm 0,05) \times 10^{3 a}$ & ND & ND & - \\
\hline & LFC 8 & $(1,30 \pm 0,20) \times 10^{4 a b}$ & ND & $(0,60 \pm 0,10) \times 10^{3 a}$ & ND & ND & - \\
\hline & LFC 9 & $(0,70 \pm 0,10) \times 10^{4 a b}$ & ND & $(0,30 \pm 0,05) \times 10^{3 a}$ & $(0,88 \pm 0,01) \times 10^{4 c}$ & $(1,14 \pm 0,14) \times 10^{4 \mathrm{e}}$ & - \\
\hline & LFC 10 & $(0,90 \pm 0,30) \times 10^{4 a b}$ & ND & $(0,43 \pm 0,13) \times 10^{4 a}$ & $(0,13 \pm 0,02) \times 10^{4 a}$ & $(1,10 \pm 0,20) \times 10^{3 \mathrm{bc}}$ & - \\
\hline \multirow{7}{*}{ 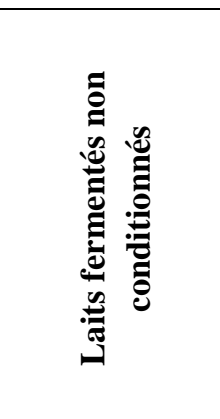 } & LFCN 1 & $(3,80 \pm 0,80) \times 10^{5 \mathrm{e}}$ & $(6,60 \pm 3,81) \times 10^{5 a}$ & $(0,42 \pm 0,08) \times 10^{6 a}$ & $(0,50 \pm 0,10) \times 10^{3 a}$ & $(0,60 \pm 0,10) \times 10^{3 a b}$ & - \\
\hline & LFCN 2 & $(0,98 \pm 0,18) \times 10^{5 a b c}$ & $(8,10 \pm 2,02) \times 10^{5 a}$ & $(1,00 \pm 0,50) \times 10^{7 b}$ & $(0,24 \pm 0,01) \times 10^{4 a b}$ & ND & - \\
\hline & $\mathrm{LFCN} 4$ & $(5,50 \pm 0,50) \times 10^{4 a b}$ & $(1,00 \pm 0,00) \times 10^{6 a}$ & $(0,24 \pm 0,44) \times 10^{6 a}$ & $(0,10 \pm 0,05) \times 10^{3 a}$ & $\mathrm{ND}$ & - \\
\hline & LFCN 5 & $(1,15 \pm 0,15) \times 10^{6 h}$ & $(5,40 \pm 4,27) \times 10^{6 b}$ & ND & $(3,92 \pm 0,58) \times 10^{4 \mathrm{e}}$ & ND & - \\
\hline & LFCN 6 & $(1,75 \pm 0,25) \times 10^{4 a b}$ & $(1,00 \pm 0,35) \times 10^{6 a}$ & ND & $(0,58 \pm 0,15) \times 10^{4 \mathrm{bc}}$ & $(1,30 \pm 0,20) \times 10^{3 \mathrm{c}}$ & - \\
\hline & LFCN 7 & $(1,72 \pm 0,18) \times 10^{6 \mathrm{i}}$ & $(7,00 \pm 5,19) \times 10^{6 b c}$ & ND & $(2,84 \pm 0,04) \times 10^{4 d}$ & $(1,10 \pm 0,40) \times 10^{3 b c}$ & - \\
\hline & LFCN 8 & $(1,03 \pm 0,03) \times 10^{6 \mathrm{~g}}$ & $(1,00 \pm 0,46) \times 10^{7 \mathrm{c}}$ & ND & $(5,76 \pm 0,76) \times 10^{4 f}$ & $(2,70 \pm 0,70) \times 10^{3 \mathrm{~d}}$ & - \\
\hline
\end{tabular}

Les valeurs suivies des lettres différentes sont statistiquement différentes $(\mathrm{P}<0,05)$.

LFC : laits fermentés conditionnés

LFNC : laits fermentés non conditionnés

ND : Non Détecté

- : Absence 


\section{DISCUSSION}

\section{Paramètres physico-chimiques}

Des variations de l'acidité et du $\mathrm{pH}$ ont été observées lorsqu'on compare les échantillons de lait fermenté non conditionné (LFNC) et les échantillons de lait fermenté conditionné (LFC). Elles peuvent être liées à la technique de fabrication utilisée pour obtenir chaque produit. En effet, au cours de la production du lait fermenté, deux types de laits sont utilisés comme matière première : le lait en poudre reconstitué ou le lait cru de vache pour les laits fermentés reconstitués et uniquement du lait cru de vache pour la production des laits fermentés non conditionnés. La durée de fermentation a eu une incidence sur la texture et la quantité d'acide lactique produit. L'acidité très élevée des échantillons de lait fermentés non conditionnés (LFNC1 à LFNC8) serait due à la durée de fermentation assez longue (en moyenne 14 heures) et à la technologie de fermentation qui se fait de façon artisanale sans aucun contrôle de souche et des quantités utilisées mais surtout sans réfrigération après fermentation pour les laits non conditionnés. Cette différence entre les produits serait également en rapport avec la flore microbienne apportée par le ferment car pour tous les échantillons, cette dernière n'est ni identifiée, ni contrôlée identifiée et le plus souvent, le lait fermenté de la veille est utilisé comme ferment. Dans la plupart des fermentations laitières, les ferments ont pour rôle de fermenter le lactose pour produire l'acide lactique. Il en résulte une baisse du $\mathrm{pH}$ et une augmentation de l'acidité Dornic au fil du temps, responsables de la coagulation de la caséine du lait et de la préservation du produit (Bourdichon et al. 2012). Ces bactéries produisent plusieurs composés aromatiques, enzymes et d'autres composés qui ont un effet profond sur la texture des produits laitiers ensemencés (Ngassam, 2007 ; Carminati et al., 2010). Le pH du lait permettant l'obtention d'une structure normale du caillé sans séparation du petit lait se situe entre 4 et 4,6 (Bourdichon, 2012; Croguennec et al., 2008) seuls les échantillons $\mathrm{LFC1}$ se retrouvent dans cette tranche. Ces $\mathrm{pH}$ faibles pourraient se justifier par les différents procédés technologiques employés par les producteurs comme a pu le démontrer Ngassam en 2007. Les différentes valeurs du $\mathrm{pH}$ obtenues sont plus acides comparées à celles de Mulonda Kakumbwa (2016) obtenues sur les laits caillés de Bukavu en République Démocratique du Congo avec des teneurs moins acides comprises entre 4,3 et 5,5. Ngassam (2007) et Dieng (2001) ont obtenu des valeurs de $\mathrm{pH}$ respectivement comprises entre 4,38 et 4,98 ; et 3,96 et 4,9 sur des laits fermentés artisanaux commercialisés au Sénégal. Par contre, Omola et al. (2014) ont obtenu des valeurs de $\mathrm{pH}$ allant de 1,44 à 5,35 sur des yaourts vendus dans la métropole de Kano au Nigeria. Les travaux effectués par Biatcho (2006) et Ngassam (2007) sur les laits caillés de Dakar ont permis d'obtenir des acidités de l'ordre de $100^{\circ} \mathrm{D}$ et $127,5^{\circ} \mathrm{D}$ respectivement.

\section{Flore microbienne}

L'abondance de la flore aérobie mésophile totale dans tous les échantillons de laits fermentés analysés pourrait s'expliquer dans la plupart des cas par l'insalubrité de l'environnement de fabrication du produit et ustensiles utilisés; le non respect des règles d'hygiène pendant la transformation de la matière première en produit. Pour les laits fermentés non conditionnés, les coups de vent, la poussière et les mouches qui se déposent sur la louche servant de mesure pendant la vente pourraient également être des sources de contamination. Millogo et al. (2018) ont pu démontrer que les récipients utilisés au cours de la fabrication du yaourt peuvent être à l'origine de la contamination du produit final.

La variation de la concentration en bactéries lactiques d'un échantillon de laits fermentés à un autre pourrait s'expliquer par la différence des procédés et techniques de fabrication. Cette variation peut également dépendre de la composition initiale du lait en bactéries lactiques, du choix du ferment (variable en fonction des producteurs), de la quantité de ferments ensemencés et par le froid qui doit être maintenu jusqu'au consommateur pour la survie des espèces 
(Ndiaye, 2002). En effet, le $\mathrm{pH}$ du lait est favorable au développement des bactéries lactiques mais leur nombre diminue au fur et à mesure que le lait fermenté vieillit (Ndiaye, 2002). L'absence de bactéries lactiques dans les échantillons de laits fermentés conditionnés (LFC 5, LFC 7, LFC 8, LFC 9, LFC 10) pourrait s'expliquer par l'utilisation d'un ferment inapproprié pour acidifier le milieu ou par le fait que certains microorganismes (bactériophages) peuvent attaquer le ferment réutilisé, ce qui pourrait altérer la flore lactique. Ces résultats sont partiellement en accord avec ceux obtenus par Ngassam (2007) sur les laits fermentés artisanaux au Sénégal où la population de bactéries lactique était de l'ordre de $10^{5}$ UFC. La flore lactique est généralement à l'origine de la production de métabolites tels que l'acide lactique et les bactériocines. La plupart ont une activité contre les pathogènes alimentaires tels que Listeria monocytogenes. En plus, L'application des bactériocines ou des souches productrices dans les aliments permet d'éviter le développement de bactéries pathogènes ou altérantes (Taale et al., 2016).

La présence des levures et des moisissures dans la plupart des échantillons serait due soit à de mauvaises conditions de stockage et de conservation, soit à une pasteurisation incomplète car ces microorganismes sont thermosensibles. Le fait de retrouver des levures dans les laits fermentés a été confirmé par Coulibaly et al. (2014) sur les yaourts vendus en Côte d'Ivoire. Selon Dieng (2002), les levures et les moisissures ont un $\mathrm{pH}$ optimal situé entre 4,5 et 6,5 donc elles peuvent parfaitement se développer dans les laits fermentés et y entraîner diverses altérations du produit. Il faut aussi noter que ces microorganismes ne sont pas gênés par l'acidité et se développent donc très facilement dans les laits fermentés. Bien que la flore fongique présente dans l'ensemble des échantillons de laits fermentés analysés ne soit pas intentionnellement introduite, leurs concentrations se rapprochent cependant des valeurs minimales requises pour le Kéfir et le Kumys (soit $10^{4}$ ).
La forte contamination des échantillons de laits fermentés par les coliformes totaux traduirait une défaillance hygiénique: soit au cours de la fabrication du produit, ce qui serait le cas pour les produits conditionnés; soit pendant l'exposition du produit aux intempéries atmosphériques et aux mains de la vendeuse. Ces résultats corroborent ceux obtenus par Biatcho (2006) et Ngassam (2007) sur les laits caillés vendus au Sénégal. Ces derniers ont dénombré des coliformes dans la plupart de leurs échantillons. Les différentes sources de contamination peuvent être: l'environnement, le personnel, les matières premières, le matériel, les techniques ou le procédé de fabrication puisque les producteurs fabriquent leurs produits dans des conditions hygiéniques douteuses.

La présence des coliformes fécaux dans certains échantillons s'expliquerait par une contamination exogène d'origine fécale. Ils sont témoins d'une hygiène défectueuse pendant ou après la transformation (Conte, 2008). La mauvaise hygiène des mains, l'environnement de fabrication, l'apport de ferment après la pasteurisation, le matériel défectueux ou contaminé constituent des sources probables de contamination (Dieng, 2001). La différence en nombre de coliformes fécaux entre les échantillons s'expliquerait par le fait que certains fabricants s'efforcent à respecter les règles d'hygiène et d'autres, par contre, ne les respectent pas. Ces résultats ne sont pas en accord avec ceux de Ngassam en 2007 sur les laits caillés du Sénégal. En effet, ses résultats ont révélé que tous les échantillons de lait caillé étaient contaminés par les coliformes fécaux avec un maximum de $1,72 \times 10^{4}$ et un minimum de $6,3 \times 10^{3}$. La présence de coliformes fécaux pourrait traduire une pasteurisation inefficace des ingrédients contaminés; une contamination après la pasteurisation par l'environnement comme l'ont démontré Niculescu et al. (2005) dans leurs travaux. En plus, certaines souches de coliformes peuvent induire des infections intestinales.

Les salmonelles n'ont pas été détectées. Si elles ont existé dans la matière première, il est possible qu'elles aient été 
détruites pendant la pasteurisation. Leur absence dans le produit final signifierait également qu'il n'y avait pas contamination au cours de sa fabrication. D'autre part, l'acidité produite par les bactéries lactiques dans les différents produits peut rendre le milieu hostile à la croissance des salmonelles. Ces résultats sont en accord avec ceux de Elham et al (2011) obtenus sur les produits fermentés du Liban, car ces derniers n'ont trouvé aucun échantillon contaminé par les salmonelles.

En définitive, parmi les échantillons de laits fermentés conditionnés, seul l'échantillon LFC6 se rapproche du yaourt malgré le fait que sa flore lactique soit faible.

\section{Conclusion}

La présente étude avait pour but d'évaluer les qualités microbiologiques des laits fermentés consommés dans la ville de Maroua. Les analyses physico-chimiques ont révélé que les laits conditionnés avaient des pH plus élevés que les laits fermentés non conditionnés, inversement, les laits fermentés non conditionnés avaient une acidité titrable très élevée. Les analyses microbiologiques ont révélé que les laits fermentés vendus à Maroua et considérés comme yaourts ne l'étaient pas, à l'exception d'un seul car ils abritaient aussi bien une flore fongique, flore lactique que quelques bactéries potentiellement pathogènes. Les laits fermentés non conditionnés avaient plus d'échantillons contaminés ( 9 sur 10) par les levures. Tous les échantillons de laits fermentés non conditionnés étaient contaminés par les coliformes totaux. Presque la moitié des échantillons analysés contenaient des coliformes fécaux. Les salmonelles, quant à elles, n'étaient présentes dans aucun des échantillons analysés. Dans la majorité, la qualité des différents laits fermentés n'est pas satisfaisante.

Il serait donc judicieux d'améliorer la qualité microbiologique des laits fermentés en vue d'assurer la santé du consommateur. Il sera nécessaire d'identifier les levures et les coliformes présents dans les laits fermentés. Il sera nécessaire d'évaluer le procédé de fabrication, de suivre l'évolution de la flore lactique des laits pendant la fabrication, et au cours de la conservation des produits obtenus.

\section{CONFLITS D'INTERETS}

Les auteurs déclarent qu'il n'existe aucun conflit d'intérêts en rapport avec ce manuscrit.

\section{CONTRIBUTION DES AUTEURS :}

MJ est l'instigatrice de ce travail, elle a conçu le projet et a supervisé l'ensemble des travaux ainsi que la rédaction et la correction du manuscrit.; BMP, AF, MYY ont travaillé au laboratoire sous la direction de MJ ; TNL et DM ont participé à la correction du manuscrit et à l'analyse des données.

\section{REMERCIEMENTS}

Nous remercions sincèrement la direction de l'Ecole Normale Supérieure de l'Université de Maroua ; tout le personnel du laboratoire de la Caisse Nationale de Prévoyance Sociale de Maroua.

\section{REFERENCES}

AFNOR (Association Française de Normalisation). 1999. Microbiologie Alimentaire: Méthodes horizontales tome 1 ( $7^{\mathfrak{e}}$ édn), Sciences et techniques agroalimentaires. AFNOR: Paris ; $663 p$.

AFNOR (Association Française de Normalisation). 2002. NF V08-059 Microbiologie des aliments Dénombrement des levures et moisissures par comptage des colonies à $25{ }^{\circ} \mathrm{C}$ - Méthode de routine. AFNOR; $272 \mathrm{p}$.

AFNOR (Association Française de Normalisation). 2009. FD V 04-035 Lait et produits laitiers - Détermination du pH. AFNOR ; 375 p.

ANSES (Agence Nationale de Sécurité Sanitaire). 2015. Avis de l'Agence nationale de sécurité sanitaire de l'alimentation, de l'environnement et du travail relatif à la définition des denrées périssables et très périssables. Saisine $\mathrm{n}^{\circ}$ 2014-SA-0061 Saisine liée n²006-SA- 
0098

https://www.anses.fr/en/system/files/BIO RISK2014sa0061.pdf. Consulté 16 Juin 2017.

Alais CH, 1985. La science du lait : principes des techniques laitières. (IVeéd), Ed SEPAIC, Paris, $814 \mathrm{p}$.

AOAC (Association of Official Analytical Chemists). 2005. Official Methods of Analysis $\left(18^{\text {th }}\right.$ Edn $)$ AOAC: Gaithersburg, MD, USA, 8; 8-25.

Biatcho NS. 2006. Appréciation de la mise en œuvre de l'hygiène dans une laiterie artisanale de Dakar «LE DIRFEL » de la récolte du lait à sa transformation en lait caillé dit «SOW PUR». Thèse de médecine de vétérinaire, Université de Dakar, Dakar, 101 p.

Bourdichon F, Casaregola S, Forrokh C, Frisva JC, Gerds ML, Hammes WP, Harnett J, Huys G, Laulund S, Ouwehand A, Powell IB, Prajapati JB, Seto Y, Schure ET, Van Boven A, Vankerckhoven V, Zgoda A, Tuijtelaars S, Hansen EB. 2012. Food fermentations: microorganisms with technological beneficial use. International Journal of Food Microbiology, 154(3): 87-97. https://doi.org/10.1016/j.ijfoodmicro.201 1.12.030.

Broutin C, Youssouf D, Mohamadou D. 2005. Guide de Bonnes Pratiques d'Hygiène. FAO ; 30 p.

Carminati D, Giraffa G, Quiberoni A, Binetti A, Suárez V, Reinheimer J. 2010. Advances and Trends in Starter Cultures for Dairy Fermentations. In Biotechnology of Lactic Acid Bacteria Novel Applications, Mozzi F (Ed). Wiley-Blackwell Publishing: USA; 393 p.

Croguennec T, Jeantet R, Brulé G. 2008. Les Fondements Physico-chimiques de la Technologie Laitière. Ed. Tec et Doc Lavoisier : Paris ; $135 \mathrm{p}$.

Conte S. 2008. Evolution des caractéristiques organoleptiques physico-chimiques et microbiologiques du lait caillé traditionnel. Thèse de Doctorat, Université de Dakar, Dakar, p. 26.

Coudeyras S, Forestier C. 2010. Microbiote et probiotiques: impact en santé humaine. Can. J. Microbiol., 56(8): 611-650. DOI 10.1139/w10-052.

Coulibaly KJ, Kouame Elogne C, Yeo A, Koffi C, Dosso M. 2015. Qualité microbiologique des produits laitiers industriels vendus à Abidjan de 2009 à 2012. Revue Bio-Africa, 14: 44-52 https://www.researchgate.net/publication /313253196 consulté le 18 juin 2018.

Dadie A, Nzebo D, Guessennd N, Dako E, Dosso M. 2010. Prévalence de Escherichia coli entéropathogènes dans le lait non pasteurisé produit à Abidjan, Côte d'Ivoire. Int. J. Biol. Chem. Sci, 4(1): 11-18. http://ajol.info/index.php/ijbcs

Dieng M. 2001. Contribution à l'étude de la qualité microbiologique des laits caillés industriels commercialisés sur le marché Dakarois. Thèse de Médecine vétérinaire, Université de Dakar, Dakar, p. 10.

Drouault S, Corthier G. 2001. Effets des bactéries lactiques ingérées avec des laits fermentés sur la santé. Veterinary Research, BioMed Central., 32(2): 101117. HAL Id: hal-00902692

Elham HS, Hussein D, Roula AR, Mabelle C. 2011. Caractérisation chimique et qualité bactériologique de produits laitiers caprins traditionnels libanais. Lebanese Science Journal, 12 (1): 21.

Essomba JE. 2000. Etude de l'hygiène de la restauration collective au Cameroun : cas du centre des œuvres universitaires de Yaoundé 1 et des cargots environnantes. Thèse de Doctorat, Université de Dakar, Dakar, $149 \mathrm{p}$.

Essomba J-M, DURY S. 2002. Consommation des produits laitiers à Ngaoundéré au Cameroun : croyances et perceptions des consommateurs et non consommateurs. Agro-PME. Yaoundé. Décembre, multigr, $22 \quad \mathrm{p}$. http://horizon.documentation.ird.fr/exldoc/pleins_textes/divers10- 
07/010038347. Consulté les 2 janvier 2017.

FDA (Food and Drug Administration). 2001. Bacteriological Analytical Manual [online] Available at: http://www.911emg.com/Ref/20Library/ 20ERG/FDA/20Bacteriological/20Analy sis .pdf . Consulté le 17 Mai 2016.

Hamad B. 2009. Contribution à l'étude de la contamination superficielle bactérienne et fongique des carcasses camelines au niveau de l'abattoir d'el-oued. Mémoire de Magister en Médecine vétérinaire, Eloued, Université Mentouri de Constantine, Algérie, p. 1-55.

Heyman M, Heuvelin É. 2006. Microorganismes probiotiques et régulation immunologique : le paradoxe. Nutr. Clin. Métabolisme, 20(2): 85-94. DOI : 10.1016/j.nupar.2006.04.003

Joly F, Coffin B, Messing B. 2007. Rôle de la flore dans les pathologies digestives (maladie de Crohn, rectocolite ulcérohémorrhagique, cancer colorectal exclus). Nutr. Clin. Métabolisme. 21(2): 89-94. DOI: 10.1056/NEJMoa067752

Millogo V, Sissao M, Ouédraogo GA. 2018. Qualité nutritionnelle et bactériologique des échantillons de quelques produits laitiers locaux de la chaîne de production au Burkina Faso. Int. J. Biol. Chem. Sci., 12(1): 244-252, DOI : https://dx.doi.org/10.4314/ijbcs.v12i1.19

Monkam N, Essomba J- M, Mabou A, Akoa J. 2002. Synthèse entreprise laitière. Projet de recherche Inco-NPE agro-alimentaire. "La performance d'un système de connexion de l'offre agricole au système de marchés surbains" Cameroun Sénégal, Guinée Bissau. Agro-PME. Avril multigr.

Mulonda Kakumbwa P. 2016. Analyse physico-chimique et microbiologique du lait caillé produit dans le groupement de miti et commercialisé dans la ville de Bukavu. Mémoire d'ingénieur, Université Evangélique en Afrique, République Démocratique du Congo. Page 32.

Ndiaye M. 2002. Contribution à l'étude de conformité de l'étiquetage de la qualité microbiologique des yaourts commercialisés à Dakar. Thèse de Doctorat, Université Cheikh AntaDiop de Dakar, Dakar, P. 11.

Niculescu N, Sabo I, Martine F. 2005. Maîtrise de la Qualité dans la Transformation Laitière au Burkina Faso / Guide de Bonnes Pratiques d'Hygiène. Ed. Polykrome: Sénégal ; 94p.

Ngassam TC. 2007. Caractérisation de la flore lactique des laits fermentés artisanaux au Sénégal : cas de la zone de Niaye. Thèse de Doctorat, Université Cheikh Anta Diop de Dakar, Dakar, p. 109.

Omola EM, Kawo AH, Shamsudden U. 2014. Physico-chemical, sensory and microbiological qualities of yoghurt brands sold in Kano metropolis, Nigeria. Bayero Journal of Pure and Applied Sciences, 7(2): 26 - 30.

Savadogo A, Traore A S. 2011. La flore microbienne et les propriétés fonctionnelles des yaourts et laits fermentés. Int. J. Biol. Chem. Sci., 5(5): 2057-2075.

DOI: http://dx.doi.org/10.4314/ijbcs.v5i5.28

Taale E, Savadogo A, Zongo C, Tapsoba F, Karou S D, Traore A S. 2016. Les peptides antimicrobiens d'origine microbienne : cas des bactériocines. Int. J. Biol. Chem. Sci. 10(1): 384-399 DOI: http://dx.doi.org/10.4314/ijbcs.v10i1.29

Vignola. 2002. Sciences et Techniques du Lait (Transformation du Lait). Ed. Presses Internationales Polytechnique Montréal ; 600 pages. 